\title{
Living with a partly amputated face, doing facial difference
}

\author{
By Gili Yaron
}

\begin{abstract}
Disability studies as an academic field has long sought to highlight the lived experiences of people with disabilities, thereby giving voice to a population that has been the object of much discourse but rarely its subject. Despite the field's engagement with various conditions, there is limited scholarly work on the personal meanings of amputation and prosthetics usage. Experiences associated with the loss of part(s) of the face, in particular, have remained uncharted. In this article, I address this lacuna by drawing on interviews with twenty affected individuals. Situating their accounts in contemporary scholarship on bodily difference within the humanities and social sciences, I demonstrate that losing part(s) of the face calls for various ways of 'doing' difference in everyday life. This empirical-philosophical analysis serves three purposes. On an empirical level, the article unpacks the everyday doing of facial difference, showing it simultaneously involves social, embodied, and material dimensions. On a practical level, this integrative understanding of facial difference complements prevalent approaches to 'disfigurement' that construe it as an individual-biomedical or psychosocial-problem. On a theoretical level the article clarifies and advances the concept of doing, which plays a key role in gender studies, phenomenology, and science and technology studies.
\end{abstract}

KEYWORDS: facial difference, disfigurement, disability, prosthesis, enactment, embodiment.

GILI YARON, senior researcher and lecturer at the Faculty of Health, Medicine and Life Sciences, Maastricht University, The Netherlands 


\section{Introduction:}

\section{Living with a partly amputated face}

Disability studies as an academic field has traditionally sought to highlight the lived experiences of people with disabilities, thereby giving voice to a population that has been the object of much discourse but rarely its subject. Despite this field's engagement with various conditions, it features limited scholarly work on the personal meanings of amputation and prosthetics usage (Murray and Forshaw 2013). Thus, research in the humanities often approaches prosthesis as an analytical trope (Jain 1999), metaphor (Mitchell and Snyder 2000), or historical phenomenon (Neumann 2010). This literature also discusses prosthetic imageries (Booher 2010; Eyler 2013), or the history of prosthetics (Ott, Serlin and Mihm 2002). The small number of studies that attend to the perspective of people affected by amputation include autoethnographic accounts (Kurzman 2001; Sobchack 2006; 2010), and empirical scholarship on experiences associated with prosthesis usage and amputation (Murray 2009; Norlyk, Martinsen and Kjaer-Petersen 2013; Mathias and Harcourt 2014).

In the case of people who lost part(s) of the face and carry a facial prosthesis, this lacuna is even more marked; research into the lived experiences associated with their particular condition is virtually non-existent. There is a growing body of literature on 'disfigurement' in general, but studies investigating this topic mostly approach it through a biomedical or psychosocial lens (Aarabi, Longaker and Gurtner 2007; Trainor, Dixon and Dixon 2009; Rumsey and Harcourt, 2004; Valente 2009). ${ }^{1}$ This focus mirrors the dualistic manner in which facial difference is commonly construed within healthcare, namely as an individual problem, that concerns either a mechanized body or a disembodied mind.

My work of the past years has sought to complement the literature on 'disfigurement' by exploring what it means to live with a partly amputated face. Analysing affected individuals' stories, my colleagues and I investigated the everyday usage of facial prosthetics (Yaron, Widdershoven and Slatman 2017), the embodiment of facial difference (Yaron et al. 2017), and the role of in/ visible difference in everyday social interactions (Yaron et al. 2018). As our work reveals, individuals who lack part(s) of the face contend with physical impairments as well as a radically altered appearance. Responding to everyday challenges associated with both, they 'do' their facial difference in various ways (Yaron et al. 2017: 303-304). The precise nature of this doing, however, remains to be unpacked. What shapes does the doing of facial difference take in the everyday life of individuals who have lost facial areas? In this article, I answer this question by situating affected individuals' accounts in contemporary scholarship on the enactment of gendered and disabled bodies within the humanities and social sciences. This empirical-philosophical approach, I conclude, expands our understanding of facial difference, while supplementing prevalent healthcare approaches to 'disfigurement' and advancing the concept of doing itself.

\section{Interrogating the everyday meaning of facial difference}

The following pages present my analysis of the lived experiences associated with the loss of part(s) of the face. Examining both old and new empirical material, I show that affected individuals' stories revolve around the ongoing doing of facial difference in various everyday settings. To unpack the distinct, interwoven shapes this doing takes, I mobilize and contrast several comparative concepts, including 'interactional accomplishment', 'gender performativity', and 'enactment'. Used to investigate everyday practices-a common concern in contemporary humanities and social sciences-this set of loosely connected approaches each highlights particular aspects of the doing of bodily difference. It is by no means my intention to provide a thorough analysis of this family of concepts; instead, I use each concept to draw out distinct ways in which facial difference is done, as featured in affected individuals' accounts. 
The analysis below draws on interviews I conducted with twenty individuals who lack part(s) of the face. These interviews formed part of an empirical-philosophical study into the everyday meaning of facial difference. Participants were eight women and twelve men, ranging in age from 42 to 84 . Each lost a nose, eye-socket (including the eye), upper cheek, or larger segments of the face. Nineteen respondents were amputated during medical treatment for head and neck cancer; one had a fireworks accident (see table 1 for an overview of the participants). Interviewees all confront various disabilities, and their appearance has altered significantly. This is due to the atypical look of the amputated facial area, as well as their usage of several more-or-less visible medical aids. Thus, participants wear conspicuous bandages, band-aids, or patches to cover the amputation site. They also carry a less noticeable silicone prosthetic device-a so-called 'facial prosthesis'-that is tailor-made to resemble the lost facial limb(s) and is attached to the face by means of medicinal glue or implanted magnets. The study received ethical clearance from the medical-ethical review board of the Dutch hospital from which I recruited interviewees (file number NL35486.031.11).

\begin{tabular}{|c|c|c|c|c|}
\hline Interview & Name \& age & Lost facial area & Cause & Aids used \\
\hline 1 & John, 65 & Nose & Cancer & $\begin{array}{l}\text { Nasal prosthesis (on implants), gauze } \\
\text { dressing, }\end{array}$ \\
\hline 2 & Timothy, 65 & Part of the left cheek & Cancer & Cheek prosthesis (glued) \\
\hline 3 & Dora, 65 & Nose & Cancer & Nasal prosthesis (glued), dressing \\
\hline 4 & Laura, 45 & Eye socket & Cancer & Orbita prosthesis (on implants), Band-Aid \\
\hline 5 & Stella, 47 & Nose & Cancer & Nasal prosthesis (glued), dressing \\
\hline 6 & Walter, 65 & Eye socket & Cancer & $\begin{array}{l}\text { Orbita prosthesis (locks into the amputa- } \\
\text { ted area) }\end{array}$ \\
\hline 7 & Arnold, 72 & Eye socket & Cancer & Orbita prosthesis (on implants), band-aid \\
\hline 8 & Ray, 66 & Right part of the nose & Cancer & $\begin{array}{l}\text { Nasal prosthesis (glued), dressing, band- } \\
\text { aid }\end{array}$ \\
\hline 9 & Lisa, 73 & Eye socket & Cancer & $\begin{array}{l}\text { Orbita prosthesis (on implants), eye } \\
\text { patch }\end{array}$ \\
\hline 10 & Bertha, 76 & Nose \& eye socket & Cancer & Complex prosthesis (glued) \\
\hline 11 & Gregory, 81 & Eye socket & Accident & Orbita prosthesis (glued) \\
\hline 12 & Oliver, 60 & Eye socket & Cancer & Orbita prosthesis (glued), eye patch \\
\hline 13 & Harry, 79 & Nose \& eye socket & Cancer & $\begin{array}{l}\text { Complex prosthesis (glued), dressing, } \\
\text { band-aid }\end{array}$ \\
\hline 14 & Audrey, 84 & Nose & Cancer & Nasal prosthesis (glued) \\
\hline 15 & Leon, 52 & Nose & Cancer & Nasal prosthesis (glued) \\
\hline 16 & Gabriel, 70 & $\begin{array}{l}\text { Nose, eye socket \& } \\
\text { part of forehead and } \\
\text { temple }\end{array}$ & Cancer & Complex prosthesis (glued), dressing \\
\hline 17 & Christine, 72 & Eye socket & Cancer & $\begin{array}{l}\text { Orbita prosthesis (glued), dressing, band- } \\
\text { aid }\end{array}$ \\
\hline 18 & Ralph, 42 & Eye socket & Cancer & Orbita prosthesis (on implants), band-aid \\
\hline 19 & Winston, 63 & Eye socket & Cancer & Orbita prosthesis (on implants), dressing \\
\hline 20 & Thelma, 57 & Eye socket & Cancer & Orbita prosthesis (glued), dressing \\
\hline
\end{tabular}

Table 1: overview of study participants 
I discussed and obtained respondents' informed consent at the start of each interview.

\section{Accomplishing difference through display and conduct}

Speaking of what it means to lose part(s) of the face, the study's participants all noted that their visible facial difference is an issue in social encounters. Particularly when going out in public while wearing a visible cover, respondents' unusual face attracts much unwanted attention:

"I walked around wearing a patch on my eye, and that generated a lot of, yes, stares and questions and comments. I wasn't anonymous anymore, I was always the centre of attention, yes, attention I found to be negative" $(\text { Ralph })^{2}$

Alluding to its power to stop others in their tracks, Stella jokingly calls the gauze dressing she sometimes wears over her missing nose an "emergency warning triangle". Interviewees dislike being the object of notice, indicating it makes them feel self-conscious, ashamed, or inferior (Yaron et al. 2018). Still, most choose to ignore covert staring, especially from children. Some actively address more offensive forms of unsolicited notice like finger pointing or giggling, for instance by pointedly staring back.

The way facial difference is handled in these social interactions resonates with what sociologists of gender Candace West, Don Zimmerman, and Sarah Fenstermaker call the 'interactional accomplishment' of difference (West and Zimmerman 1987; West and Fenstermaker 1995; 2002). Social categories like gender, race, and class, they argue, do not refer to static phenomena that precede social exchanges. Instead, members of society accomplish such categories during everyday interactions. Gender, for instance, is "a routine, methodical and recurrent accomplishment (...), an achieved property of situated conduct" (West and Zimmerman 1987: 126). Social categories are accomplished through the repetition of typical acts, displays, and speech patterns that are associated with, for example, femininity, blackness, or 'poshness'. ${ }^{3}$ Achieving gender, race, and class lies at the very heart of human sociality; The doing of difference structures each and every social situation (e.g. sex-segregated public bathrooms, sports, dating practices). Social actors continuously evaluate whether they themselves and others establish relevant social identities appropriately. To accomplish difference, therefore, necessarily means to stand the risk of assessment. Such assessment has real consequences: individuals are held accountable for how they accomplish gender, race, or class, and penalized if they fail to do so appropriately (West and Zimmerman 1987).

The concept of interactional accomplishment underlines how visible facial difference is achieved through display and conduct in the daily life of the participants in my study. Others' unwanted attention in effect holds respondents accountable for deviating from the norm prescribing 'proper' appearance. Since they are (implicitly) expected to answer to others' curiosity, such attention casts interviewees as less entitled to the 'civil inattention' accorded to those with a typical exterior (Goffman 1967). Indeed, blatant staring, giggling, and finger pointing-forms of what disability scholar Rosemarie Garland-Thomson calls 'uncivil attention' (2006; 2009)-clearly penalize respondents for their unusual appearance. Facial difference can therefore be said to be done in interaction.

By using facial prosthetic device, participants regularly manage to avoid others' attention:

\footnotetext{
"Yes, I can just tell that since I have gotten my prosthesis, that less people really stare (...). I could tell instantly, immediately in that first week already. Yes, [it's different] than when you're wearing a Band-Aid. (...). People don't notice [my eye] as much" (Laura, wears an eye-socket prosthesis)
}

Since it recovers facial completeness, this device may restore users' ability to 'pass as normal' (Yaron, Widdershoven and Slatman 2017). To pass, interviewees must ensure the prosthesis 
'fits' their face and vice versa. Nevertheless, others regularly realize the device is in fact artificial. This mostly happens when respondents stand in close proximity to others for prolonged periods of time: "Sometimes at the cash register, because you'd be standing close to people, it happens that the 'nose' is suddenly discovered. [Their] gaze is like, 'wait a minute..."' (Stella). Participants mostly ignore such instances of discovery, although they sometimes address them. Their efforts to pass as normal, as well as their responses to being discovered, form another way of doing difference through display and conduct.

However, the accomplishment of gender, race, or class is distinct from the doing of facial difference in one significant regard. As anthropologist Marcel Mauss' concept of 'body techniques' illustrates, people signal their membership in particular groups (e.g. societies, professions, generations) by adopting sometimes subtle though very much recognizable stylistic 'signatures': particular ways of moving, positioning the body, gesturing, etcetera (Mauss 1979). In the case of disability, however, many typical ways of appearing and moving are associated with impairment and the usage of assistive devices. These are therefore not the product of a socialization process ${ }^{4}$. Indeed, rather than exhibiting characteristic mannerisms, many people with disabilities in fact develop body techniques aimed at suppressing or concealing their difference in favour of looking 'normal'. Likewise, respondents' attempts to pass as normal are oriented towards appearing ordinary. Their ways of doing facial difference, therefore, revolve around endeavouring to accomplish normalcy rather than difference.

\section{Performing identity via discourse}

Next to discussing display and conduct, interviewees also shared stories on how their facial difference is spoken about. Thus, others regularly ask about or comment on participants' unusual appearance. Respondents mostly view polite queries as intrusive though relatively benign. Some questions and comments, however, are not so innocent.
Discussing others' reactions to the bandage she initially wore to cover her missing nose, Dora says: "[T]otal strangers (...) would say (...) 'Well you've had some rap on the nose, haven't you?' (...) and 'You have a weird noggin'". Other offensive utterances respondents mention include: 'How do you find the courage to go on?', 'If I were you, l'd just put an end to it all', or 'Can't [doctors] do something about your face?'. When others discover the prosthetic to be a fake, they may also remark upon the device ('It's such a clever solution!') or ask to touch it.

To understand the role of language in how facial difference is done, I turn to feminist philosopher Judith Butler's notion of 'performativity' (Butler 2007; 2011). Departing from an analysis of drag, Butler's work challenges common distinctions between sex and gender. The material body, in her account, exists beyond meaning-making but can never be approached outside of it: since human experience is necessarily mediated by signs, we can have no direct access to some type of 'pre-' or 'extradiscursive' bodily reality (Butler 2007: $\mathrm{xx}$ ). Human beings, for Butler, "come into being" in language: it is only through their assimilation into the order of signification that they emerge as subjects (idem: 8). The sexed body is therefore always already gendered: immediately after birth (and often before), the new-born's body is classified as either female or male on the basis of anatomical features (e.g. genitalia) and thereby received into a signification system. In Butler's words: "medical interpellation (...) shifts an infant from an 'it' to a 'she' or a 'he,' and in that naming, the girl is 'girled,' brought into the domain of language and kinship" (Butler 2007: xvii).

With Michel Foucault, Butler argues that subjects are produced as such through the repetition of patterns of expression. ${ }^{5}$ Individuals are neither the authors nor the origins of the statements they make, but draw these from established discourses-repositories of historically rooted linguistic forms (e.g. phrases, jargons, plotlines) that organize communication. When subjects reproduce specific types of speech, they in fact assert their and others' membership in a distinct social group. Such utterances are 'performative', for Butler, in that they establishing speakers (and addressees) 
as particular subjects. 'Hello, I'm Mrs. Smith, nice to meet you,' for instance, positions the speaker as a married woman. Importantly, discourses are inherently normative, constituting certain individuals as members of a devalued social category.

When others bring up participants' unusual appearance, from a Butlerian perspective, they in fact perform the visibly different person as an extraordinary, deviant spectacle. Specifically, such utterances constitute respondents as objects of ridicule, suffering victims, incapable of having a worthwhile (social) life, or in need of medical intervention. This type of everyday language reflects stereotypical meaning commonly attributed to atypical bodies. Blending into a "disfigurement imagery" (Talley 2014: 18, 28-30), representations of facial variance generally depict it as a pitiful state requiring a medical 'fix'. Using Butler, it becomes possible to understand both these representations and the everyday utterances that echo and instantiate them as part of a normative discourse on visible facial difference-a discourse that produces affected individuals as tragic (or, alternatively, heroic) victims-regardless of the fact most live rather ordinary lives.

As Butler indicates, subjects also perform their own identity through language. Indeed, next to utterances made by others, interviewees do their visible difference verbally as well. This type of talk may occur when they reprimand others for staring, pointing, or giggling:

"[T]here were those girls (...), teenagers, right. And they obviously noticed something and started laughing constantly, 'hahaha', you know. And then at some point I said, 'You should ask your mom to teach you some manners maybe and not gape at people" (Dora)

Another type of talk follows more benign types of notice. When asked politely about their facial difference, respondents may explain about their condition. Taking control of and defusing a mutually awkward situation, they assure their interlocutor that they handle everything 'just fine'. Similarly, participants may opt for anticipatory self-disclosure in situations in which others are likely to discover their face is partly prosthetic ${ }^{6}$ :

"[M]y wife [and I] gave (...) a marriage course [to recently married couples from Church], and then (...) I would always just say it. And, well it's over then. You can tell that people [are] no longer as fixated on your face anymore (...). Because l'd openly laid it out to be spoken about" (Timothy)

Finally, respondents may also joke about their artificial facial limb with others who know about it, like (grand)children, colleagues or friends. Oliver, a high school teacher, discusses a pun he sometimes uses in conversation with students:

"Well, if someone needs to be taken aside [for] detention, [I'd say, 'let's have] a talk under three eyes.' And then you see them, you see someone all blushing" (Oliver, carries an eye-socket prosthesis)

Explanations and jokes, in particular, acknowledge interviewees' difference while simultaneously normalizing it. Such talk construes the person in question as being at ease with their uncommon face. It also establishes that they are a fundamentally ordinary human being, despite their uncommon appearance. Moreover: explanations and jokes indicate the facially different person deserves the same civility accorded to other members of society.

Participants responses to unwanted attention can in fact be read as forms of Butlerian resistance. Adopting a Derridean strand into her thought, Butler asserts that the repetition of established patterns of discourse is in fact a type of citation. Since every reiteration necessarily alters the meaning of the utterance it echoes, citation opens up spaces for difference. Speakers therefore do not only emulate discursive patterns, but may also improvise upon them, for instance through parody or out-of-context usage. Subversive citations, particularly, may undermine and resist oppressive categorizations. By emphasizing their fundamental normalcy and humanity, interviewees upset 
conventional representations of 'disfigurement' as a pitiful, exceptional, and dehumanizing state. By jokingly giving new meanings to old phrases, they 'own' their difference while resisting being reduced to it. Through these verbal forms of doing difference, participants defy being 'othered' while encouraging interlocutors to approach them as ordinary, fully human actors, worthy of equal regard.

\section{Doing lived embodiment}

The concepts of interactional accomplishment and performativity both highlight how bodies are done through various types of signification (display, conduct, language) that structure sociality. Nevertheless, it remains unclear how the experiencing body figures in these accounts. As Carrie Noland writes: "Butler has a meagre account of both embodiment and interoception. (...) [S] he neglects to theorize the performing body's proprioceptive, kinesthetic, even affective experience of moving in prescribed ways" (Noland 2009: 171). This criticism, I argue, equally applies for West and her collaborators. To be sure, the stories of the participants in my study illustrate that doing bodily difference encompasses a marked embodied dimension.

In the interviews, respondents spoke of various bodily experiences. All contend with sensory impairments, including the (partial) loss of smell, skin sensitivity, or sight. Discussing her reduced visual field after losing her eye, Lisa says: "While cycling [with friends] (...) if they ride on the left, we can't converse and I can't see them very well". Other interviewees report problems with navigating steps, pouring drinks, stacking boxes, tasting food, feeling a kiss, or smelling hazardous fumes. In addition, many also deal with unpleasant bodily sensations in and around the amputation site, such as chronic pain, phantom itching, or irritation. Others remain conscious of the prosthesis: "I'm constantly aware of that thing. I feel it, for sure, that it's loose, a bit" (Leon). Finally, anatomical changes to participants' sinuses may affect the trajectory of air and mucus. This results in either hindering dryness or leakage. As Christine, who lost her eye, says: "[L]uckily I don't often come down with the cold, but l've had it happen sometimes (...) [that mucus] would leak from underneath the prosthesis. (...) [T]hat was a disaster". Consequently, participants can no longer use their body as they did before; their formerly taken for granted capacity to pursue everyday activities has been disrupted. Gradually adjusting, interviewees develop an array of new bodily habits to anticipate, avoid, and manage disruptions (Yaron et al. 2017). By finding a more careful way of walking, a new manner of kissing, or strategies to handle unpleasant sensations, they in fact learn to do their body differently.

This bodily doing recalls phenomenologist Iris Marion Young's writing on gender in On female body experience: 'Throwing like a girl' and other essay (2005). As Young demonstrates, the physical im/possibilities afforded by female bodies call for distinctive ways of enacting the body. ${ }^{7}$ Pregnancy involves adjusting to one's growing girth and may cause tiredness and nausea. Breasts may be sensitive, produce mother's milk, or hamper one's ability to engage in sports (Rodrigues 2018: 266-268). Menstruation may be accompanied by cramps and requires the management of flow. Women, in other words, live through the inevitable materiality of their bodies. Young's account therefore opens up a perspective on bodily doing that goes beyond signification to encompass lived experience. Similarly, the $\mathrm{im} /$ possibilities afforded by a partly amputated face mean that affected individuals need to develop new ways to perceive, sense, and move as they relearn their altered body and world.

But Young's account of female embodiment also explores women's experiences in relation to the socio-cultural meanings accorded to their bodies. Youngs analysis of feminine motility, for example, argues that the cautious inhibition characterizing many women's movements in fact reflects internalized gendered norms (Young 2005; Weiss 2015). As she writes: "The more a girl assumes her status as feminine, the more she takes herself to be fragile and immobile, and the more she actively enacts her own body inhibition" (Young 2005: 44). Young's take on gender emphasizes 
that such norms affect not only the way in which women present their social identity, but also how they use and experience their bodies. Gender is shaped both through the meanings inscribed upon the feminine body, and the material vicissitudes of that body. Indeed, Young's originality lies in the fact she successfully weaves together these two seemingly disparate dimensions of embodiment, while stressing the fundamental materiality of both.

The doing of facial difference, too, simultaneously encompasses both dimensions. Participants' stories about spilling tea due to a limited visual field, failing to sense a kiss on the cheek, or having mucus run down the corner of an eye, all speak to practical-functional concerns. But such incidents also testify of social ones: the embarrassments arising from bodily mishaps.

And indeed, the (possibility of) social censure respondents confront affects how they use and experience their body. Unwanted attentionas well as the work involved in anticipating, avoiding and managing it-effectively constrains their ability to inhabit public spaces:

"In the past (...) I would go out and grab a sandwich or a milk carton or whatever. And I would pull on my pants in a flash and run outside (...). Now I would have to wait an hour and a half lgetting the prosthesis to fit just right], before I can leave the house" (Leon)

Interviewees also restrain their facial movements to ensure others do not discover the prosthesis is a fake. Many keep the affected side of their face averted, chew or yawn carefully to prevent the device from gaping, or control their blinking to ensure their prosthetic and organic eyes 'match'.

Following the amputation, the face effectively takes on a new existential meaning. As the locus of potential physical as well as social perils, this body part has become much more 'present' in participants' consciousness. Such presence disrupts their ability to focus on everyday activities. What is more: being rendered a liability, the face has come to require ongoing monitoring and management.
Enacting artefacts, environments, and assistive devices

Young's writing on embodiment regularly alludes to the ways in which physical artefacts and environments tie in with the enactment of female bodies. After all, body, object, and world come into being simultaneously according to the phenomenological tradition in which she operates. Nevertheless, since Young focuses on bodily experience, her analyses provide little explicit attention to the materialities involved in bodily doing. Similarly, both West and her collaborators and Butler centre the meaning of things, rather than their materiality. As participants' stories demonstrate, however, the doing of facial difference has a solid material dimension.

As established, respondents use medical aids such as bandages, eye patches, and prostheses. The materiality of these aids affects the doing of facial difference. For instance, Laura's prosthetic eye-socket irritates her skin. She therefore reserves the device for outings, preferring her more comfortable bandage when staying at home. Others' comfort is also involved. Thus, the inflexibility and coldness of Stella's artificial nose prompted her to develop new ways to kiss loved ones. The fact passing as normal requires that the prosthesis look like a natural part of the face, also calls for material work (Yaron, Slatman and Widdershoven 2017). This may involve daily maintenance to ensure the device remains in good shape, but also various creative adjustments:

"The edges [of my nasal prosthesis] (...) start becoming wavy [after a while] and then it gets, well, less nice-looking (...), so at some point I will make a small cut-out [points to the lower corner of his 'nostril'], and then I can pinch [the two parts] neatly together, and it stays put better" (Gabriel, wears a complex prosthesis that replaces his nose, left eye, and parts of his temple)

In addition, interviewees repeatedly reported using other types of objects to help them handle the changes imposed by the amputation. They install 
mirrors on their bicycle to compensate for limited visual field, use crayons to mark off distance while stacking boxes, or wear shawls when going outside in winter to protect their now-vulnerable sinuses. Respondents also use various artefacts to increase their chances of passing as normal: "To somewhat disguise that eye I choose really flashy glasses [that] block [of the edges of the prosthesis] from view" (Thelma). Others use a sunlamp or theatre make-up to ensure their skin tone and the device's hue keep matching, despite changes to their skin tone. These everyday objects, then, can also be said to do facial difference.

Environmental conditions play a role in the doing of facial difference as well. Wind and rain may blow away or drench appliances:

"I [keep on] my gauze dressing, if I don't have to leave the house. But not outside, no, no. (...) I mean, you can't walk in the rain with it. You can't walk in the wind. It's really for home" (Stella)

Cold weather can cause pain in the amputated area of the face. It may also redden the skin and cause silicone to shrink-both of which make the prosthesis' artificiality more evident, thereby thwarting participants' efforts to pass as normal. The lay-out of public spaces may also affect passing. Checkouts, busses, and cafés afford prolonged encounters between strangers that invite unwanted attention. By contrast, busy streets encourage moving along quickly, which helps respondents pass. Similarly, neon lighting makes the prosthesis seem fake, while daylight and shade are more forgiving.

The concept of 'enactment' as developed by science and technology scholar Annemarie Mol in The Body Multiple: Ontology in Medical Practice (2002), can help account for this material doing. Mol leverages this concept to investigate how medical knowledge is mobilized in hospital settings, through various practices involving human and non-human actors alike. The term 'enactment', she maintains, captures the fundamental materiality of practices, their dynamic, emergent character, and the way they assemble disparate elements (Mol 2002: 32, 37-43).
But how do patients enact disease? 'Enacting bodies, Embodied Action: The example of Hypoglycaemia' (Mol and Law 2004) investigates how hypoglycaemia is done in the everyday life of affected individuals ${ }^{8}$. Some ways of enacting this condition revolve around knowing it, for instance by measuring blood glucose or learning to sense an oncoming drop in sugar levels ('hypo'). Such knowledge, Mol stresses, is geared towards anticipating and counteracting manifestations of the condition: "In the daily lives of people with diabetes, hypoglycaemia is something [patients] know about, but the point of their dealings with it is not to gather knowledge but to intervene" (Mol and Law 2004: 49). Interventions involve 'incorporating' various objects. For instance, patients use medical instruments to monitor blood glucose or inject insulin, and eat snacks to offset hypo's. Interventions may also involve 'excorporating' the body by "shifting out" or transferring some of its actions outwards (idem: 53). Thus, patients keep snacks in strategic locations to be able to deal with dropping blood glucose. Enacting disease, the body incorporates its material environment, while also excorporating into it.

Similarly, individuals who lack part(s) of the face incorporate assistive devices. They may also incorporate everyday objects such as shawls or sunglasses. And they incorporate their environment, for instance by seeking out natural light, shadow, and corners while avoiding neon lights and central locations. In addition, respondents also excorporate their facial difference, adjusting their physical surroundings (e.g. installing bicycle mirrors, marking off distance with crayons) to accommodate their altered face. These incorporations and excorporations are all part of the doing of facial limb absence, part of a newly-developed relationship between the altered, extended body and its various contexts. As Mol observes, bodies have semi-permeable boundaries: inside and outside, self and other, organism and artefact are not so easily distinguished. Moreover, participants' efforts to accomplish a good fit between their face and prosthesis illustrate that the body is an essentially unfinished, open project, requiring supplementation as well as continuous work to produce 
and maintain its integrity. As Mol and Law put it: "[k]eeping yourself whole is one of the tasks of life. It is not a given, but must be achieved, both beneath the skin and beyond, in practice" (2004: 57, emphasis in the original).

\section{Conclusions: Doing facial difference}

Zooming in on the lived experiences of individuals who lost part(s) of the face, this article has sought to expand our understanding of facial difference and thereby contribute to scholarship on amputation, prosthetics, and facial difference. The amputation of facial areas, as demonstrated above, signals a major shift in the formerly taken for granted relationship between participants' body and its social and material environment. Respondents' altered, extended face no longer quite fits their world. All the while, that world proves not-so welcoming for bodies that diverge from the norm. As Garland-Thomson argues, disability is a relational phenomenon, the function of 'misfits' between the impaired body's physical make-up and contexts that fail to accommodate bodily variance (Garland-Thomson 2011; Weiss 2015). This lack of fit, as interviewees' stories reveal, elicits various ways of doing facial difference.

To unpack the multiple dimensions involved in this doing, I interpreted my empirical findings against the background of four distinct approaches to the enactment of bodily difference. West, Zimmerman, and Fenstermaker's concept of interactional accomplishment provided insight in the role of display and conduct in social interactions between the visibly different person and others, while underscoring that these revolve around accountability and penalization. Butler's notion of performativity highlighted the role of language in these interactions. It also shed light on normative discourses informing how facial difference is done in everyday social exchanges, as well as on ways in which affected individuals resist and upend prevalent meanings attached to 'disfigurement'. Importantly, both these approaches helped establish that it is normalcy rather than deviance which is at stake in the doing of facial difference.
Young's analysis of sensory-motor experience made for a deeper understanding of the embodied dimensions of the doing of facial difference. Mol's concept of enactment, finally, drew attention to the material affordances of medical aids, everyday artefacts, and environments, as these tie in with the doing of facial difference. In this sense, the sociology of gender, phenomenology, and science and technology studies provide complementary insights into this doing.

As these approaches reveal, facial difference is done in shifting arrangements in which bodies, norms, meanings, artefacts, and environments meet. The active doing of facial difference, however, is always also a passive 'being done'. While self and other both do facial difference in interaction, this doing is co-determined by interactional norms as well as common representations of 'disfigurement'. Moreover: although they actively adapt to their altered face, individuals with facial difference are nevertheless constrained by that face's im/possibilities, and those of aids, everyday objects, and settings ${ }^{9}$. As Lisa Käll concludes in her discussion of Butler's concept of performativity, voluntarism and determinism may go hand in hand (Käll 2015).

Understanding what it means to lose part(s) of the face, requires simultaneously addressing the social, embodied, and material dimensions of facial difference. Indeed, it is only by taking these three perspectives in concert, that it becomes possible to arrive at an integrative account of facial difference. Arguably, this point holds for bodily variance in general. Such an integrative account has much to offer healthcare. After all, adequately informing, caring for, and supporting patients, requires a firm grasp of how they experience and respond to their altered-body-in-context.

An integrative account of facial difference may thereby complement how 'disfigurement' is currently approached in healthcare, namely as an either biomedical or a psycho-social problem. This outlook is prevalent in research as well as in practice, including preoperative counselling, care and support, and rehabilitation. Here, I will restrict myself to a pertinent example I encountered in the hospital in the form of the 'disfigurement' protocol 
offering postoperative nursing guidelines for patient care. This protocol focuses primarily on the physical healing process after the amputation surgery, but nurses are also encouraged to monitor signs of 'psychological dissociation' following the patient's first confrontation with a mirror. 'Body image' is mentioned in this context, but this term is far removed from its original meaning, which indicates "the physical, social and imaginative aspects of bodily experience" (Rodrigues 2018: 259). In the clinic, 'body image' narrowly refers to the patient's psychic attitude ('cognitions') regarding their changed appearance.

In this way, the 'disfigurement' protocol evokes the patient as an organic machine containing a disembodied mind. As disability theorist Margrit Shildrick argues in her phenomenologically-informed study of heart transplantation, such dualism is fundamentally reductionist (Shildrick et al. 2009). By upholding it, healthcare fails to recognize and address the inherent entanglement of body and psyche, as well as the existential impact of bodily changes. As a result, patients' attempts to make sense of their radically altered bodily being are relegated to the background. All the while, the social is curiously absent in the protocol; formal care is restricted to the individual patient's body (and to a lesser extent, mind). And indeed, not only do patients' embodiment and social embeddedness disappear from view, but also their ongoing daily negotiations with the materialities of medical aids, everyday artefacts, and physical environments ${ }^{10}$.

Shildrick calls for a critical turn in feminist bioethics (Shildrick 2008). Insights from poststructuralism and postmodernism, she argues, can help the field move beyond traditional women's issues and the ethics of care. The "postconventional canon" may thereby offer a truly critical alternative to mainstream bioethics (Shildrick 2008: 34). Like her earlier work, Shildrick's heart transplants project again demonstrates the 'leakiness' of the body: the fact that bodiesand therefore selves-are inherently vulnerable, open-ended, fragmented, and saturated with difference. Her goal is to "dispense with the notion of a core self that persists unchanged" (idem: 38). Like Mol, Shildrick contends that bodily integrity is an achievement rather than a pre-given condition.

These insights are not only vital for bioethics, but can help to counter healthcare's dualistic thrust. How they may be translated to practice, however, remains to be unpacked. Shildrick proposes adopting a phenomenologically informed framework in patient care, which will enable accepting and recognizing alterity, vulnerability, and concorporeality (Poole et al. 2009; Shildrick 2008). Her suggestions nevertheless remain somewhat vague in terms of practical applications. Moreover, as my focus on materiality illustrates, patients' accounts reveal a fuller range of experiences than those strictly associated with the body. Future work could further explore the clinical implications of focusing on the altered body-in-context.

Analysing empirical data on facial difference against the background of theories on the enactment does not only supplement current approaches to 'disfigurement'. My findings also serve to clarify and advance the notion of doing itself. By mobilizing and contrasting the concepts of interactional accomplishment, performativity and enactment, this paper has provided a clearer sense of their scope, revealing strengths, divergences, and blind spots. This empirical-philosophical approach thereby further develops a set of interrelated though distinct concepts that plays a key role in current debates within the sociology of gender, phenomenology, and science and technology studies. In this way, theory can inform practice, while empirical findings may enrichen conceptual work. 


\section{Notes}

1 For one recent exception, see (Martindale and Fisher 2019) on embodied identity and narrative meaning making in facial disfigurement. As the authors note, scholarship on the ethics of facial transplants offers some theoretical explorations of facial difference and identity, but empirical studies remain scarce.

2 Respondents' names are all pseudonyms.

3 According to West, Zimmerman, and Fenstermaker, individuals accomplish gender, race, and class simultaneously: the ways in which these categories take shape together therefore result in highly specific, situated, and shifting configurations of difference.

4 To somewhat qualify this claim: One notable exception is found in people with a hearing impairment who embrace Deaf culture. Another is so-called 'expressive' prostheses. which are not designed to resemble the lost limb, but rather to openly express the prothesis' artificiality-often through a cyborg-like style (Hall and Orzada 2013).

5 Butler sees not only language but also expression and signification as performative. Since this section focuses on verbal ways of doing facial difference, I use Butler's account to highlight speech.

6 For an overview of interviewees' strategies in handling unsolicited notice, see (Yaron et al. 2018).

7 Although she repeatedly uses the term enactment to describe typically feminine motility, Young's usage of this terms seems almost incidental: she never systematically develops this notion as an analytical concept.

8 Interestingly, Mol and Law's account neglects to discuss appearance-related concerns and stigma in the enactment of hypoglycaemia. This oversight underlines the importance of the comprehensive approach presented in this paper.

9 For a similar argument with regard to women's agency in the context of 'disfiguring' breast cancer, see (De Boer and Slatman 2018).

10 Many nurses undoubtedly address these issues informally on the basis of experience and intuition. However, the setup of healthcare practices remains deeply dualistic. Moreover, professionals at present lack training, guidelines, and time to provide more integrative forms of care.

\section{References}

Aarabi, S., Longaker, M. T. and Gurtner, G. C. 2007. Hypertrophic scar formation following burns and trauma: New approaches to treatment. PLoS Medicine. 4(9), 1464-1470. doi: 10.1371/journal. pmed.0040234.

De Boer, M. and Slatman, J. 2018. The mediated breast: Technology, agency, and breast cancer. Human Studies. 41(2), 275-292. doi: 10.1007/s10746-017-9445-5.

Booher, A. K. 2010. Docile bodies, supercrips, and the plays of prosthetics. IJFAB: International Journal of Feminist Approaches to Bioethics. 3(2), 63-89. doi: 10.3138/ijfab.3.2.63.

Butler, J. 2007. Gender trouble: Feminism and the subversion of identity. London and New York: Routledge.

Butler, J. 2011. Bodies that matter: On the discursive limits of 'sex'. London and New York: Routledge.

Eyler, J. R. 2013. Disability and Prosthesis in L. Frank Baum's The Wonderful Wizard of Oz. Children's Literature Association Quarterly. 38(3), 319-334. doi: 10.1353/chq.2013.0042.

Garland-Thomson, R. 2006. Ways of staring. Journal of Visual Culture. 5(2), 173-192. doi:

$10.1177 / 1470412906066907$.

Garland-Thomson, R. 2009. Staring: How we look. New York: Oxford University Press.

Garland-Thomson, R. 2011. Misfits: A feminist materialist disability concept. Hypatia. 26(3), 591-609. doi: 10.1111/j.1527-2001.2011.01206.x. 
Goffman, E. 1967. Interaction ritual: Essays in face-to-face behavior. New York: Doubleday

Hall, M. L. and Orzada, B. T. 2013, Expressive Prostheses: Meaning and Significance. The Journal of Design, Creative Process \& the Fashion Industry. 5(1), 9-32. doi: 10.2752/175693813X13559997788682.

Jain, L. 1999. The prosthetic imagination: Enabling and disabling the prosthesis trope. Science, Technology \& Human Values. 24(1), 31-54. doi: 10.1177/016224399902400103.

Käll, L. F. 2015. A path between voluntarism and determinism: Tracing elements of phenomenology in Judith Butler's account of performativity. Lambda Nordica: Tidskrift om homosexualitet. 20(2-3), 23-48.

Kurzman, S. L. 2001. Presence and prosthesis: A response to Nelson and Wright. Cultural Anthropology. 16(3), 374-387. doi: 10.1525/can.2001.16.3.374.

Martindale, A-M. and Fisher, P. 2019. Disrupted faces, disrupted identities? Embodiment, life stories and acuired facial 'disfigurement'. Sociology of Health and Illness. 41(8), 1503-1519. doi: 10.1111/14679566.12973.

Mathias, Z. and Harcourt, D. 2014. Dating and intimate relationships of women with below-knee amputation: an exploratory study. Disability and Rehabilitation. 36(5), 395-402. doi: 10.3109/09638288.2013.797509.

Mauss, M. 1979. Sociology and Psychology: Essays. London: Routledge and Kegan Paul.

Mitchell, D. T. and Snyder, S. L. 2000. Narrative prosthesis: Disability and the dependencies of discourse. Ann Arbor: University of Michigan Press.

Mol, A. 2002. The body multiple: Ontology in medical practice. Durham: Duke University Press.

Mol, A. and Law, J. 2004. Embodied action, enacted bodies: The example of hypoglycaemia. Body \& Society. 10(2-3), 43-62. doi: 10.22394/0869-5377-2017-1-233-259.

Murray, C. D. 2009. Being like everybody else: The personal meanings of being a prosthesis user. Disability and Rehabilitation. 31(7), 573-581.

Murray, C. D. and Forshaw, M. J. 2013. The experience of amputation and prosthesis use for adults: a metasynthesis. Disability and Rehabilitation. 35(14), 1133-1142. doi: 10.3109/09638288.2012.723790.

Neumann, B. 2010. Being prosthetic in the First World War and Weimar Germany. Body \& Society. 16(3), 93-126. doi: 10.1177/1357034X10373403.

Noland, C. 2009. Agency and embodiment: Performing gestures/producing culture. Cambride Massachutts/Londen: Harvard University Press.

Norlyk, A., Martinsen, B. and Kjaer-Petersen, K. 2013. Living with clipped wings-Patients' experience of losing a leg. International Journal of Qualitative Studies on Health and Well-being. 8(1). doi: 10.3402/ qhw.v8i0.21891.

Ott, K., Serlin, D. and Mihm, S. 2002. Artificial parts, practical lives: Modern histories of prosthetics. New York: NYU Press.

Poole, J., Shildrick, M., McKeever, P., Abbey, S. and Ross, H. 2009. 'You might not feel like yourself': Heart Transplants, Identity and Ethics. In: Murray, S. and Holmes, D, eds. Critical Interventions in the Ethics of Healthcare: Challenging the Principle of Autonomy in Bioethics. Farnham: Ashgate, 33-45.

Rodrigues, S. 2018. To learn the world again: Examining the impact of elective breast surgery on body schema. Human Studies. 41(2), 255-273. doi: 10.1007/s10746-018-9462-z.

Rumsey, N. and Harcourt, D. 2004. Body image and disfigurement: Issues and interventions, Body image. 1(1), 83-97. doi: 10.1016/S1740-1445(03)00005-6.

Shildrick, M. 2008. The Critical Turn in Feminist Bioethics: The Case of Heart Transplantation. International Journal of Feminist Approaches to Bioethics. 1(1): 28-47.

Shildrick, M., McKeever, P., Abbey, S., Poole, j. and Ross, H. 2009. Troubling Dimensions of Heart Transplantation. Medical Humanities (BMJ Supplement). 35(1): 35-38.

Sobchack, V. 2006. A leg to stand on: Prosthetics, metaphor, and materiality. In: Smith, M. and Morra, J., 
eds. The prosthetic impulse: From a posthuman present to a biological future. Cambridge: MIT Press, 17-41.

Sobchack, V. 2010. Living a 'phantom limb': On the phenomenology of bodily integrity. Body \& Society. 16(3), 51-67. doi: 10.1177/1357034X10373407.

Talley, H. L. 2014. Saving face: Disfigurement and the politics of appearance. New York and London: New York University Press.

Trainor, P. A., Dixon, J. and Dixon, M. J. 2009. Treacher Collins syndrome: Etiology, pathogenesis and prevention. European Journal of Human Genetics. 17(3), 275-283. doi: 10.1038/ejhg.2008.221.

Valente, S. M. 2009. Visual disfigurement and depression. Plastic Surgical Nursing. 29(1), 10-16. doi: 10.1097/01.PSN.0000347718.75285.23.

Weiss, G. 2015. The normal, the natural, and the normative: A Merleau-Pontian legacy to feminist theory, critical race theory, and disability studies. Continental Philosophy Review. 48(1), 77-93. doi: 10.1007/ s11007-014-9316-y.

West, C. and Fenstermaker, S. 1995. Doing difference. Gender \& Society. 9(1), 8-37. doi: $10.1177 / 089124395009001002$.

West, C. and Fenstermaker, S. 2002. Doing gender, doing difference: Inequality, power, and institutional change. New York: Routledge.

West, C. and Zimmerman, D. H. 1987. Doing gender. Gender and Society. 1(2), 125-151.

Yaron, G., Meershoek, A., Widdershoven, G., Van den Brekenl, M and Slatman, J. 2017. Facing a disruptive face: Embodiment in the everyday experiences of "disfigured" individuals. Human Studies. 40(2), 285307. doi: 10.1007/s10746-017-9426-8.

Yaron, G., Meershoek, M., Widdershoven, G. and Slatman, J. 2018. Recognizing difference: In/visibility in the everyday life of individuals with facial limb absence. Disability and Society. 33(5), 743-762. doi: 10.1080/09687599.2018.1454300.

Yaron, G., Widdershoven, G. and Slatman, J. 2017. Recovering a "disfigured" face: Cosmesis in the everyday use of facial prostheses. Techne: Research in Philosophy and Technology. 21(1), 1-23. doi: 10.5840/techne2016121659.

Young, I. M. 2005. On female body experience: 'Throwing like a girl' and other essays. New York: Oxford University Press. 